\title{
Germanica
}

allemande au XXe siècle

\section{L'émulation du monde ancien : Altneuland de Theodor Herzl}

Das aemulative Überbieten der alten Welt : Altneuland von Theodor Herzl

Till R. Kuhnle

\section{OpenEdition}

\section{Journals}

Édition électronique

URL : http://journals.openedition.org/germanica/2076

DOI : 10.4000/germanica.2076

ISSN : $2107-0784$

Éditeur

Université de Lille

Édition imprimée

Date de publication : 31 décembre 2002

Pagination : 143-157

ISBN : 9782913857100

ISSN : 0984-2632

Référence électronique

Till R. Kuhnle, «L'émulation du monde ancien : Altneuland de Theodor Herzl », Germanica [En ligne], 31 | 2002, mis en ligne le 21 mars 2013, consulté le 06 octobre 2020. URL : http://

journals.openedition.org/germanica/2076 ; DOI : https://doi.org/10.4000/germanica.2076

Ce document a été généré automatiquement le 6 octobre 2020.

(c) Tous droits réservés 


\title{
L'émulation du monde ancien : Altneuland de Theodor Herzl
}

\author{
Das aemulative Überbieten der alten Welt : Altneuland von Theodor Herzl
}

\author{
Till R. Kuhnle
}

Aujourd'hui, au milieu des cahots de l'autobus, allant à Währing, le titre de mon roman sur Sion m'est venu : Altneuland. Évoque le nom de l' Altneuschul de Prague. (Herzl, le 30 août 1889)

\section{Le progrès, ce grand malentendu}

À l'origine du malentendu, il y a l'idée de Progrès. Avec la plupart de leurs contemporains, les Juifs nouvellement émancipés tenaient pour acquises l'inscription de la Raison dans l'histoire. Cet optimisme temporel réduisait la barbarie à un archaïsme, et faisait du Mal la forme violente de la bêtise ou de l'erreur. (Finkielkraut, 89)

1 Le rejet de tout archaïsme par les idées des Lumières et l'espérance eschatologique qui s'ensuit sont représentés, selon Alain Finkielkraut, par la Zauberflöte de Mozart qui met en scène la lutte finale entre la superstition et la raison. Cette espérance a subi une crise profonde lors de la révolution industrielle quand les progrès des sciences et de la technologie commençaient à s'accélérer sans que le genre humain ait connu ce mouvement vers la perfection tant attendu par l'optimisme de certains philosophes des Lumières comme Condorcet ou G.E. Lessing : tout en éloignant une grande partie des populations des exploits de la technique moderne, la nouvelle société bourgeoise et capitaliste avait créé de nouveaux clivages au sein des sociétés occidentales. Pour maints penseurs juifs de la fin du XIX ${ }^{e}$ siècle, les Lumières mettaient en cause le juif en « kaftan » des ghettos qui était considéré comme étant à l'origine des réticences du côté des non-juifs antisémites ainsi que des juifs assimilés et embourgeoisés qui adoptaient l'idée de la perfectibilité du genre humain. L'image du juif en « kaftan » était associée à 
une attitude archaïque qui allait à l'encontre de la Raison et dont le juif "éclairé » voulait s'émanciper pour assurer son assimilation.

2 Selon Theodor Herzl, le juif européen appartient à une classe moyenne exclue d'une vraie participation économique et sociale. Ainsi marginalisé, il attire la haine des antisémites qui ramènent cette position sociale à sa mauvaise foi :

Dans la population, l'antisémitisme croît de jour en jour, d'heure en heure : il ne cessera pas de croître, car ses causes continuent d'exister et ne peuvent être écartées. La cause lointaine en est la disparition de la faculté d'assimilation, intervenue au Moyen Âge ; la cause proche de notre surproduction en intelligences moyennes, qui ne trouvent de débouchés normaux vers le haut ni vers le bas. Vers le bas, nous sommes prolétarisés en révolutionnaires et fournissons ainsi les sousofficiers des partis révolutionnaires, alors qu'en même temps, vers le haut, notre pouvoir financier ne cesse de grandir. (Judenstaat, fr. 40, all. 31)

Dans l'introduction à Der Judenstaat (fr. L'État juif ou L'État des juifs, cf. Klein : Note), ce manifeste du sionisme rédigé en 1896 à la veille du Congrès mondial du Sionisme à Bâle de 1897, Theodor Herzl revendique les idées des Lumières françaises et de l'Aufklärung allemande qu'il maintient contre la crise que l'idée du progrès connaît vers cette fin de siècle.

Un autre penseur juif d'origine allemande, Max Nordau, dresse l'inventaire pessimiste ; d'après son essai au ton crépusculaire - dont le titre sera transformé en arme idéologique par la propagande cynique du nazisme : Entartung (« la dégénérescence ») les sociétés européennes sont épuisées : leur civilisation n'offre plus qu'un tableau de symptômes psychopathologiques parfaitement traduit par l'œuvre de Richard Wagner (Nordau, 5sq, 314). Les notions de "décadence » et de "dégénérescence » marquent le revers de toute philosophie de l'Histoire qui pose une société de justes comme « eschaton». Et cela au moment où l'humanité dispose des moyens techniques pour réaliser ce rêve.

Dans cette situation, les sionistes sont amenés à voir une chance pour les juifs en Europe. L'histoire des peuples parmi lesquels les juifs ont été jetés par la diaspora n'est point leur histoire à eux : le moment paraît venu pour former une nation juive. Pour Sartre, la collectivité juive est même « la moins historique de toutes les sociétés car elle ne peut garder mémoire que d'un long martyre, c'est-à-dire d'une longue passivité » (Sartre 81). Par conséquent, il n'y a rien d'étonnant à ce qu'un intellectuel juif ne partage point une philosophie de l'Histoire, voire une eschatologie, basées sur les fondements matériels et idéologiques d'une société dont il se voit rejeté. Si, par contre, Herzl se réfère à l'histoire de son peuple, c'est tout d'abord pour resserrer les rangs du mouvement sioniste. En évoquant la patrie historique des juifs, il fait appel à une histoire révolue mais toujours présente en tant qu'impératif moral. Ainsi il exclut catégoriquement l'alternative d'un état juif en Argentine.

6 Herzl est loin d'abandonner l'idée de la perfectibilité ; par contre, ce n'est plus le genre humain qu'il a en vue, mais la civilisation en tant que telle. L'analyse du développement politique et social du XIX ${ }^{e}$ siècle l'amène à la conclusion que ce mouvement est un long processus, à condition qu'on sache tirer profit des exploits de la science :

Je crois à l'ascension de l'homme vers des degrés de civilisation toujours plus élevés; seulement cette ascension est d'une désespérante lenteur. Si nous devions attendre jusqu'à ce que l'esprit de la moyenne des hommes atteigne une tolérance semblable à celle de Lessing dans Nathan le Sage, non seulement notre vie y passerait, mais aussi celle de nos fils, de nos petits-fils et de nos arrière petit-fils. 
C'est là que l'esprit du siècle vient nous secourir par une voie imprévue.

(Judenstaat, fr. 21sq, all. 14)

7 Herzl reste sceptique par rapport à la nature humaine et s'oppose explicitement aux idées de Rousseau : "Vouloir améliorer les conditions actuelles par la bonté humaine équivaut purement et simplement à écrire une utopie » (Judenstaat, fr. 41, all. 32 ; cf. id. all. 82). Herzl esquisse dans Der Judenstaat le programme d'une société juive en Palestine basée sur un système coopératif et mutualiste respectant la propriété : il revendique alors un réalisme technique et économique.

\section{Altneuland}

«Si vous le voulez, ce ne sera pas un rêve » - c'est le slogan mis en exergue du roman Altneuland (fr. Terre ancienne - terre nouvelle ou Le Pays ancien-nouveau), paru en 1902, dans lequel Herzl développe le projet sioniste sous forme d'une fiction politique dont l'action débute en 1903. Le héros est un jeune juif viennois: Friedrich Löwenberg. Partageant le sort de maints juifs, ce docteur en droit se trouve en marge de la société viennoise : "Les nouvelles générations émigraient donc en masse vers les professions libérales. Ils constituaient ce pitoyable surplus de gens instruits ne trouvant pas d'emploi, et en même temps inaptes à un mode de vie modeste, impossibles à caser dans l'administration, comme leur collègues chrétiens » (Altneuland, fr. 34sq, all. 3). Ce désœuvrement imposé par la société et son amour déçu pour une fille bourgeoise plongent Friedrich dans une mélancolie profonde. Tout en le totalisant par sa condition de juif, il est atteint du mal du siècle, cette mélancolie qui envahit les sociétés européennes à la fin du XIX ${ }^{e}$ siècle : l'avenir lui paraît barré. Dans son désespoir, il répond à l'annonce d'un certain "Nobody " qui cherche quelqu'un de prêt à tout abandonner et à se livrer à une expérience inouïe: l'excentrique Kingscourt, un misanthrope riche, veut quitter la civilisation pour vivre sur une île. Le jeune Friedrich doit l'accompagner, à condition qu'il prononce un serment qui l'oblige de rester aux côtés de Kingscourt jusqu'à la fin de ses jours. Friedrich accepte, mais avant de partir il donne encore tout l'argent qu'il a reçu de son nouveau maître à une pauvre famille juive en train de mourir de faim. Leur voyage vers leur retraite les mène d'abord en Palestine où ils ne rencontrent que de la détresse.

Puis le roman connaît un blanc: les vingt années de leur existence dans un paradis tropical. En 1923, quand ils décident de retourner pour une dernière fois en Europe, ils font encore escale en Palestine. Et à leur grand étonnement le pays a complètement changé. Là où régnaient la détresse et le désespoir, une nouvelle société prospère. Leur surprise est d'autant plus grande quand Friedrich est reconnu dans le port de Tel Aviv : c'est David Littwack, le fils de la famille sauvée par la générosité de celui qui a voulu rompre avec l'humanité. Friedrich est noyé sous la reconnaissance - même la maison de David et de sa famille porte son nom: Friedrichsheim. Avec David, les amis commencent à découvrir la terre cultivée par les juifs d'Europe. Ils doivent apprendre les progrès que l'humanité avait faits depuis qu'ils l'avaient fuie, des progrès dont le noyau est la Palestine, le Sion des Juifs du monde entier : «Altneuland, pays anciennouveau, murmura Friedrich ».

10 «Précisement, dit David Littwak, grave et ému. Nous avons édifié une nouvelle société sur notre chère vieille terre » (Altneuland, fr. 101, all. 73sq). En peu de temps, les juifs émigrés d'Europe et des autres continents ont créé une civilisation pacifique en 
mettant en œuvre les exploits techniques et culturels de la vieille Europe. Leur état, la "Nouvelle Société", est le fruit d'une entreprise gigantesque basée sur une administration modèle. Bien que la nature humaine ne puisse être changée, la "Nouvelle Société » a su en tirer le meilleur parti et ainsi minimiser les conflits sabotant toute vie en communauté. Les activités bénévoles et économiques vont de pair dans ce système mutualiste. La fusion d'un paternalisme avec les principes d'une économie moderne est le fondement politique d'un système qui veut échapper à la politique "politicienne" en choisissant ses dirigeants parmi les plus désintéressés, c'est-à-dire parmi ceux qui ont déjà fait preuve d'esprit civique sans jamais aspirer à se lancer dans une carrière politique. Et la notion d'État est remplacée par celle d'une "société de citoyens" sans armée (Altneuland, fr. 115sq, all. 89sq). La "Nouvelle Société » d'Altneuland s'entend comme une réponse - réponse bien bourgeoise - au défi lancé par le socialisme :

Croyez-moi, notre mutualisme ne nous a pas appauvris en fortes personnalités, il nous a enrichis. L'individu n'est ni écrasé par les meules du capitalisme, ni décapité par le nivellement socialiste. Nous reconnaissons et respectons l'individu, comme nous respectons et protégeons sa base économique, la propriété privée. (Altneuland fr. 127, all. 102)

11 Vers la fin du roman, David est élu président de la "Nouvelle Société» à laquelle il a initié ses amis. Convaincu de la société nouvelle et tombé amoureux de la sœur de David, Friedrich souhaite rester en Palestine. Bien qu'encouragé par la famille de David, il doit d'abord y renoncer parce qu'il est lié à Kingscourt par son serment. Mais entretemps, celui-ci n'a pas moins fléchi sous les discours maïeutiques de leurs hôtes: il réussit à surmonter ses réticences toujours latentes contre les juifs. Même lui, ce misanthrope, trouvera sa place dans une société qui accueille les non-juifs, les musulmans comme les chrétiens, et les invite à contribuer à ce que le rêve sioniste soit réalisé : "Toutes les actions des hommes ont commencé par le rêve et plus tard redeviennent rêve » - sur cette phrase finit le roman de Herzl.

C'est Ernst Bloch qui, dans Das Prinzip Hoffnung (Le Principe espérance), insistera sur les fondements idéologiques de ce rêve :

La civilisation toute entière du tournant du siècle y [Der Judenstaat/L'État juif] fait l'objet d'un transfert de fonds : «Lorsque nous quitterons à nouveau l'Égypte, nous n'oublierons pas d'emporter le meilleur ». De cette manière, si les juifs le veulent, " la fable deviendra réalité », et un roman utopique de 1900, intitulé Terre ancienne, Terre nouvelle, poursuivait la peinture du pays progressiste bourgeois, où l'on s'était installé dans sa propre tente, au milieu de ses propres vignes, comme jadis chez soi, en Europe, mais maintenant entre soi. En raison du changement minime apporté au système économique dans l'état juif modèle, cette utopie n'était pas installé dans un avenir très éloigné : elle se déclare compte rendu de l'année 1920. (Bloch, fr. 192, all. 704)

\section{Utopie et idéologie : stratégies de ré-écriture}

Le sociologue allemand Karl Mannheim distingue la conscience utopique de la conscience idéologique : « Utopisch ist ein Bewußtsein, das sich mit dem es umgebenden 'Sein' nicht in Deckung befindet " (Mannheim, 169). La conscience utopique ne se confond pas à l'être qu'il transgresse pour le faire éclater en faveur d'une nouvelle organisation des données sociales et économiques. Dans le cours de son Histoire, l'homme aspirait toujours à cet ailleurs qui s'exprime dans la pensée utopique, tout en posant par une 
conscience idéologique, conscience en parfait accord avec son être, le moule concret pour créer le cadre réel de sa vie (Mannheim, 169) - bref : la civilisation.

En partant des catégories proposées par Mannheim, il est possible de distinguer deux stratégies d'écriture, notamment la stratégie idéologique et la stratégie utopique. Vus de près, les écrits de Herzl se révèlent, selon les catégories de Mannheim, à la fois idéologiques et utopiques. Leur côté "utopique» réside dans la nouvelle mission attribuée au juifs, mais ils n'impliquent pas moins des re-lectures ou ré-écritures (cf. Barthes: SZ, 10) idéologiques d'une pensée transmise par des types de discours hétérogènes, tout en constituant une cohérence de «transtextualité » (Genette, 7) : en tant que textes clos ils se posent comme des énoncés marqués par une vraisemblance qui ne laisse entrevoir aucune faille idéologique par laquelle ils s'avèreraient utopiques. Le roman Altneuland est "vraisemblable" dans la mesure où il s'inscrit dans un discours dont, selon Kristeva, le «'faire-semblant-d'être-une-vérité-objective' est reconnu » (Kristeva, 150). Il en résulte la cohérence idéologique de son discours. Ainsi Herzl peut revendiquer, dans Der Judenstaat, un «réalisme" qui repose sur une modernité considérée comme illimitée dans ses possibilités :

Déjà le mot 'impossible' semble avoir disparu du vocabulaire de la technique. Si un homme du siècle passé revenait sur terre, il trouverait dans notre vie toutes sortes de manifestations magiques inconvenables. Partout où nous les modernes apparaissons avec les moyens dont nous disposons, nous transformons le désert en jardin. On construit aujourd'hui des villes en autant d'années qu'il fallait autrefois de siècles. L'exemple américain le montre abondamment. L'éloignement n'est plus un obstacle. L'esprit moderne recèle des richesses incalculables; chaque jour, cette richesse augmente. Des centaines de milliers de cerveaux travaillent, cherchent partout et ce qui vient d'être découvert appartient immédiatement à la terre entière. (Judenstaat, fr. 109, all. 98)

La stratégie rhétorique est évidente : les exploits de la technique moderne se portent garant de la cohérence des arguments par leur soumission à une topique, ce qui permet d'exclure le mot «impossible » qui pourrait être remplacé ici par « in-vraisemblable ». À l'instar du locus amoenus de la littérature bucolique qui est devenu un «signe universel, celui de la Nature » (Barthes : Rhétorique, 309), la modernité est devenue un « signe universel » - celui de la culture humaine : le topos de la « modernité » réconcilie l'empirisme avec l'où-topos, le non-lieu, cette Palestine à conquérir. Cette conception d'une modernité transformée en topos, conception bien bourgeoise et l'essence même de tout "modernisme", a permis à Herzl de renoncer à tout recours théologique comme par exemple dans la déclaration fondatrice du judaïsme réformé américain, présentée en 1885 à Pittsburg (cf. Haddad, 68) - pour réconcilier la tradition messianique et les exploits du monde «moderne». Ainsi Herzl peut proclamer «vraisemblable» la transition de la fiction à la réalité : «Il ne dépend donc que des juifs que cet essai politique ne reste provisoirement qu'une fiction politique [all. Staatsroman] » (Judenstaat, fr. 18, all. 12). Herzl se réfère ici explicitement à un genre littéraire : le Staatsroman. Les Staatsromane sont tout d'abord les écrits, dans la tradition d'Utopia de Thomas More, qui ébauchent des sociétés nouvelles sans pourtant revendiquer la possibilité de leur accomplissement dans l'immédiat. Bien que le roman Altneuland annonce déjà les doutes sur la validité du projet de rassembler tous les juifs sous la bannière du sionisme, il n'exprime pas moins la conviction qu'il suffit de donner une terre aux juifs pour transformer cette « utopie » en politique : "Ainsi, l'utopie de Herzl était-elle accessible dans l'immédiat et se dessinait-elle sur un fond de 
démocratie capitaliste; enracinée dans le sol, la seule chose qu'elle ne possède pas encore, elle ne poursuivait aucune chimère » (Bloch, fr. 192, all. 704).

Les exploits économiques, politiques et sociaux, les inventions tout court, s'entendent plutôt dans le sens de l'inventio rhétorique, la reprise d'arguments et de formes (ici : de procédés techniques) déjà connus :

L'inventio renvoie moins à une invention (des arguments) qu'à une découverte : tout existe déjà, il faut seulement le retrouver : c'est une notion plus « extractive » que «créative ». Ceci est corroboré par la désignation d'un «lieu » (la Topique), d'où l'on peut extraire les arguments et où il faut les ramener: l'inventio est un cheminement (via argumentorum). (Barthes : Rhétorique 293sq)

Dans tous les détails, l'épure d'une nouvelle société juive en Palestine puise ses arguments dans la topique de la modernité, au moins d'une modernité telle qu'elle est représentée par l'idéologie bourgeoise du $\mathrm{XIX}^{\mathrm{e}}$ siècle. C'est ainsi que le juriste Herzl s'approche des possibilités techniques: elles sont pour lui tout d'abord des topoi et point des analyses effectuées par un ingénieur. Mais il ne veut pas se limiter à une pure imitatio: son inventio - sa vision des innovations techniques réalisées en Palestine s'entend comme émulation. En rhétorique, l'aemulatio signifie la totalisation de la via argumentorum dans le but de la surpasser vers une perfection invitant, à son tour, à l' imitation. Ainsi, le message d'Altneuland peut être résumé de la manière suivante : il ne s'agit ni d'imiter ni de transgresser la civilisation occidentale vers une utopie, mais de la surpasser en la ré-inventant par aemulatio. Dans ce Staatsroman, la rhétorique l'emporte sur la fiction, dans la mesure où la société nouvelle est présentée à travers les explications dont le discours prend souvent un tournant maïeutique afin d'initier le héros. Bref: l'« utopie " Altneuland est "vraisemblable». Aussi dans ses éléments pourtant rares - d'écriture romanesque, à tout moment, la fiction s'enchaîne sur le "monde vécu» (all. "Lebenswelt») par le lecteur. Tout en totalisant l'idéologie bourgeoise du XIx ${ }^{e}$ siècle, Herzl fait preuve d'une vision particulière de la destinée des juifs européens: l'idée de l'assimilation aux sociétés européennes, qui ne signifiait qu'une pure imitation de leurs valeurs, est remplacée par celle de l'émulation dans leur marche vers l'accomplissement de la civilisation.

\section{Digression : les « colons » de Jules Verne}

18 Un grand «visionnaire » d'une telle approche non-historique et rhétorique à l'idée de la civilisation est Jules Verne. Ses romans sont des extrapolations minutieuses des possibilités offertes par les exploits des sciences. Le capitaine Nemo parcourt les 20 mille lieues sous les mers à bord de son sous-marin Nautilus. Pour construire ce sous-marin, il a appliqué les connaissances de son époque. Mais le Nautilus est une entreprise prométhéenne : Nemo dresse l'inventaire du monde sous-marin pour lui seul sans avoir l'intention de le communiquer. Ce nouveau Prométhée a totalisé le savoir du XIx siècle pour une idée fixe qui s'avérera meurtrière. Il en est de même avec Robur le conquérant, qui explique que l'humanité n'est pas encore prête à tirer profit de son aéronef: elle ne s'en servirait qu'en tant que moyen de destruction. Dans l'univers vernien, Nemo et Robur ont pour contrepartie le personnage de l'ingénieur Cyrus Smith qui amène les naufragés de L'̂̂le mystérieuse à former une communauté idéale ou celui de l'ingénieur humanitaire qui fonde avec sa part des 500 millions de la Bégum une ville idéale, Franceville. La "vision» du monde de Cyrus Smith exige un inventaire des 
connaissances acquises par l'humanité qui attendent d'être exploitées - pour assurer la survie des naufragés avec les moyens modestes dont ils disposent.

Genette en conclut qu'on y "voit - catastrophe finale mise à part - la robinsonnade virer massivement à l'utopie » (Genette, 431). Il est à souligner que Genette oublie que après que lî̂le et le Nautile ont été engloutis par la mer - les naufragés de L'Île mystérieuse baptisée «Lincoln Island» emploient le trésor qui leur a été confié par le capitaine Nemo pour fonder une novelle colonie en Amérique : « New Lincoln ». On est incliné à dire que les colons de "New Lincoln» ont obtenu cette terre refusée jusqu'alors aux juifs. Et c'est encore avec Genette qu'on peut souligner cette vraisemblance "rhétorique » des écrits de Verne - ainsi que de ceux de Herzl - dans la ré-écriture des utopies :

[...] le mouvement habituel de la transposition diégétique est un mouvement de translation (temporelle, géographique, sociale) proximisante : l'hypertexte [sc. le texte en transformant un autre] transpose la diégèse [sc. la narration] de son hypotexte [le texte transformé - ici : Robinson Crusoe de Defoe] pour la rapprocher et pour l'actualiser aux yeux de son propre public. (Genette, 431)

La comparaison d'Altneuland avec les œuvres de Jules Verne s'impose pour maintes raisons: d'abord il y a cette approche rhétorique des exploits de la technologie moderne dont ce-dernier fait preuve avec les nombreuses dialogues didactiques insérés dans ses romans; puis Herzl et Verne se rejoignent dans leur visions d'une nouvelle société patriarcale appliquant ces exploits pour améliorer les conditions de vie de ses membres. Sans doute, Jules Verne aurait pu souscrire à la devise suivante qui exprime la relation entre les ouvriers et leurs patrons dans la «Nouvelle Société»: «[...] c'est une relation patriarcale, mais concrétisée dans les formes les plus modernes" (Altneuland, fr. 128, all. 103).

Herzl et Verne partagent aussi le rejet d'une mélancolie destructrice. Dans L'Île mystérieuse, le capitaine Nemo rejoint l'humanité en léguant à Cyrus Smith et à ses amis un trésor qui leur permettra, après avoir été sauvés du cataclysme, de recommencer en Amérique. Mutatis mutandis, on peut retrouver l'impératif éthique de Verne dans l'histoire de Kingscourt et de Friedrich : l'un est poussé par des expériences décevantes à la misanthropie, l'autre est amené par un chagrin d'amour à considérer définitivement sa vie comme dépourvue de sens, et tous deux renoncent à mettre leurs capacités au service du bien commun. Mais en donnant l'argent à Friedrich qui en profite pour sauver la famille juive en détresse, Kingscourt a contribué d'une manière indirecte à ce que David puisse devenir le nouveau « père » de la société nouvelle. A la fin, le juif Friedrich ainsi que le "goy " Kingscourt ressortent de leur mélancolie pour se rendre utile à la «Nouvelle Société». C'est en restant en Palestine, qu'ils assument désormais leur responsabilité. Ils sont le contraire du capitaine Nemo transformé par la mélancolie en Prométhée déchaîné ; et ils respectent aussi l'avertissement de Robur déclarant que l'humanité doit être prête à savoir tirer profit des exploits des sciences qui risquent de devenir des instruments de destruction ou - comme dans Paris au $\mathrm{XX}^{e}$ siècle, ce roman anticipant par maints éléments les anti-utopies comme Brave New World de Huxley - une fin en soi pour condamner ainsi les hommes réifiés à la mélancolie. Il n'y a de vrai progrès qu'au service de l'humanité. 


\section{Le rejet d'une rupture apocalyptique}

22 Le refus de la notion d'utopie par Herzl repose sur un scepticisme anthropologique, c'est-à-dire sur le rejet de la croyance naïve de Rousseau en la nature humaine ainsi que de son scepticisme par rapport à la civilisation - et, par conséquent, d'une vision eschatologique ayant comme fondement l'idée de la perfectibilité de l'espèce humaine. Et pourtant, Altneuland reprend aussi un motif de la littérature u-chronique : par des circonstances merveilleuse, le héros se réveille des années, voire des siècles, après s'être endormi et découvre les exploits que l'humanité a faits depuis. Dans son roman L'An 2440 de 1774, Sébastien Mercier a entraîné son héros à la découverte d'un Paris nouveau, entièrement réorganisé selon les idées des lumières pour apprendre à la fin que cette ère nouvelle avait été précédée par la chute "apocalyptique» de l'Ancien régime (Mercier, 293sq). Mais la ville nouvelle, cette cité des lumières, ne fut qu'un rêve ! Environ 150 ans après le rêveur de Mercier, le héros d'Altneuland revient dans un pays civilisé après avoir vécu 20 ans aux côtés de son ami Kingscourt sur une île déserte.

La «Nouvelle Société " à été réalisée par la communauté «la moins historique » (Sartre) qui seule en était capable parce qu'elle n'avait pas à porter le fardeau du passé - sans pourtant renier cette histoire lointaine toujours présente dans sa latence :

Sans doute nous avons, nous aussi, renoué avec le passé, et il le fallait - la terre ancienne, le peuple ancien - mais les institutions ont été rajeunies. Le peuples qui ont porté sur le dos une histoire ininterrompue étaient nantis de charges contractées par leur pères. (Altneuland, fr. 114, all. 88)

Comme le souligne Ernst Bloch, le théoricien marxiste - et pourtant très théologique de l'apocalypse, le sionisme de Herzl repose sur la volonté de reproduire, dans des conditions idéales, un environnement bien européen mais plus complet et tourné vers l'avenir. Par conséquent, Herzl récuse tout millénarisme radical qui - à l'instar des millénarismes chrétiens et judaïques ou de la révolution marxiste - annonce une rupture imminente : l'anéantissement apocalyptique du vieux monde pour faire naître sur ses décombres une ère (messianique) nouvelle (cf. Haddad, 95). Toutefois, il n'abandonne pas ce rêve dans lequel convergent tous ces millénarismes: celui d'un avenir illimité dans ses possibilités dont l'avènement est imminent.

Naguère, on discutait sans fin de l'État futur, les uns d'une manière fumeuse, certains avec mépris, d'autres encore avec fureur. Aux yeux des gens soi-disant pratiques, il était suprêmement ridicule de chercher à esquisser l'État à venir. Ils oubliaient que nous vivons le futur à chaque instant, car aujourd'hui n'est rien d'autre que l'avenir d'hier. On envisageait l'impossible État de demain sur les ruines improbables des institutions d'aujourd'hui. C'est-à-dire une sorte de fin du monde, que seul un poltron peut envisager. D'abord le chaos, puis l'ordre nouveau dont il est douteux qu'il fût meilleur que l'ancien. [...] L'ancien n'a pas nécessairement à disparaître d'un coup, pour permettre le surgissement du neuf. [...] Depuis que j'ai vu ici qu'on peut bâtir un nouvel ordre des choses avec des matériaux anciens, je ne crois plus ni à la destruction complète ni au renouvellement intégral des institutions. Je crois [...] à une construction graduelle de la société. Je crois aussi qu'elle ne se fait jamais selon un plan, mais à mesure des besoins. La nécessité est son architecte. (Altneuland fr. 348, all. 334)

L'eschatologie apocalyptique est dénoncée comme le fruit de la peur, comme l'apogée d'une mélancolie destructrice (cf. Blumenberg, 78 ; Kuhnle 1999). En même temps, le traumatisme de l'homme dépassé par les progrès déchaînés de la technologie moderne 
est paré par l'idée d'une évolution qui repose sur les besoins de l'homme entrainant les progrès nécessaires dont partait, entre autres, ce "visionnaire " éminemment conservateur qu'était Jules Verne. L'Histoire est bannie à jamais ! Et les habitants de la nouvelle Palestine ont créé leur état Altneuland - comme les naufragés de L'T̂le mystérieuse ou les «colons» de Franceville ont fondé leurs «colonies»: ils étaient libérés des contraintes du monde ancien. Mais plus que les «colons» de Verne, les habitants d'Altneuland sont conscients d'une mission universelle :

Nous [les Juifs] seuls étions en mesure de créer cette nouvelle Société et ce foyer de civilisation. Une chose découle de l'autre, et seul notre destin pouvait servir d'accoucheur. Nos souffrances morales, notre expérience économique ont nécessairement contribué à l'œuvre. (Altneuland, fr. 118sq, all. 93)

C'est désormais le rôle des juifs de mettre en œuvre la réalisation des idées des Lumières ainsi que de l'économie moderne et de former «l'avant-poste de la civilisation contre la barbarie » (Judenstaat, fr. 47, all. 37). Le rôle attribué par Herzl au peuple juif est celui de l'avant-garde de l'humanité :

Le monde sera libre par notre liberté, enrichi de notre richesse, agrandi de notre grandeur. Et ce que nous tenterons là-bas pour notre propre prospérité aura des effets puissants et heureux pour le bien-être de l'humanité tout entière. (Judenstaat, fr. 115, all. 104).

\section{Épilogue : I'humanisme hébraïque et la ré-écriture d'un blanc}

27 Samuel Beckett s'en prend à l'ambiguïté de cette conception du Messie dans les plus célèbres de ses pièces: En attendant Godot et Fin de partie. Dans la première, il fait tourner au ridicule une attitude passive qui limite tout espoir au maintien d'un statu quo pourtant peu humain ; dans la deuxième il confronte à sa vision post-apocalyptique une vieille histoire drôle qui apparaît comme un regard nostalgique sur l'humanisme juif (Beckett, 36-38) : c'est le tailleur juif qui, en se révélant incapable d'achever le pantalon d'un gentleman anglais, crée une métaphore satirique résumant le destin d'un humanisme écrasé par le cours de l'Histoire qui enlève le mouvement du progrès des mains d'une humanité impuissante. La technologie moderne qui, selon Herzl et la tradition (bourgeoise) des Lumières, était censée être au service du développement du genre humain, et qui, selon Bloch, s'est transformée en " Todesmittel », en instrument de mort, est dorénavant un danger imminent pour toute entreprise utopique. Hans Jonas, un des grands philosophes juifs de langue allemande, s'avère le critique le plus retentissant de la pensée utopique qu'il dénonce comme totalitaire a priori. Mais dans son livre Das Prinzip Verantwortung, un règlement de comptes au vitriol avec Das Prinzip Hoffnung d'Ernst Bloch, Herzl n'est même pas mentionné, ainsi que toutes les tentatives de révision de la pensée utopique sous l'égide d'un nouvel humanisme judaïque.

Selon Hermann Cohen, un adversaire du sionisme, l'idée d'une nation juive allait à l'encontre de la revalorisation de l'idéal messianique (Cohen, 421) étroitement liée à une réévaluation de l'Histoire (Cohen, 307sq). Reprise et développée par maints penseurs judaïques comme Sholem ou Benjamin, cette position est devenue un instrument critique dénonçant tout totalitarisme (cf. Kuhnle : 1998, 115-117) :

Mettant à profit les moyens techniques les plus modernes, la «religion politique » de l'État-nation a marqué de son empreinte l'histoire $d u x^{e}$ siècle : deux guerres mondiales, généralisation des «états d'exception » totalitaires. Hostile à l'État, la 
philosophie messianique/libertaire des écrivains juifs allemands se situe aux antipodes de ce « messianisme étatique » triomphant. (Löwy, 251)

D'une certaine manière, on est porté à croire que les écrits sionistes de Herzl qui, en tant que "Staatsromane", se révèlent plus idéologiques qu'utopiques (ou «messianiques ») sont bannis par le verdict «politically not correct» (cf. Klein : Essai, 186) - verdict affirmé non par un autodafé, mais par le silence. Autrement dit : il s'agit de la « ré-écriture » d'un blanc, car les écrits de Herzl, pourtant mineurs comme textes littéraires, sont toujours présents. Ce blanc se fait ressentir, par exemple, dans un essai comme celui de Meschonnic sur L'Utopie du juif:

Un des mythes mobilisateurs du sionisme étant celui de la régénération, il présupposait lui-même le motif antisémite de la dégénérescence. Dégénérescence, mot de Max Nordau, titre de son livre de 1893, contre la poésie symboliste en particulier, et préparant involontairement le terme ultérieur d'art dégénéré. Le sionisme préconisait, contre un long avilissement, une régénération «par le travail et le contact de la Nature» [Lindenberg]. (Meschonnic, $327 \mathrm{sq}$ )

Pourquoi Nordau et pas Herzl ? On se pose bien la question étant donné que ce dernier a essayé d'inspirer un nouvel élan à cette fin de siècle qu'il voulait revaloriser notamment «par le travail et le contact de la nature » ainsi que par la technologie moderne. Serait-ce le fruit d'un antisémitisme de gauche toujours aussi présent, dans sa latence, que l'antisémitisme tout court? ou de l'absence des traditions théologiques et culturelles du judaïsme dans les épures d'un état sioniste? Pour Shmuel Trigano qui évite aussi le nom de Herzl - le sionisme de 1948 « a peu à voir avec le sionisme fondateur. C'est une idéologie désespérée. Elle est devenue l'idéologie de l'exil» (Trigano, 48).

31 Lors du centenaire du mouvement sioniste, Bernard-Henri Lévy note : «Que reste-t-il, demandent ses héritiers [du sionisme], du message de Theodor Herzl ? Et la plupart de répondre - et ils ont évidemment raison : l'obligation, à la fois stratégique et morale, de faire la paix avec les Palestiniens » (Lévy, 241). Dans cette notice, Lévy rappelle à la fois le côté idéologique et le côté utopique de la pensée de Herzl et de ses héritiers - tout en se limitant à une situation politique précise: le premier exige un accord avec l'adversaire pour garantir l'avenir de l'état d'Israël (l'obligation «stratégique»), le deuxième souligne l'impératif éthique transcendant toute idéologie (l'obligation «morale ») vers cette "régénération » dont parle Meschonnic, régénération dans le sens d'un humanisme judaïque universel. Le discours pathétique de Trigano souligne aussi cet universalisme inné à l'idée du « Grand Retour » :

La vocation ultime de la judéité est d'appeler les peuples à cette communauté universelle dont leur cœur vibrera en Jérusalem. « De Sion, chacun dira qu'il y est né » (Ps. 87, 4). Et peut-être l'épanchement de la parole préparera-t-elle la convergence vers Jérusalem d'une communauté de tous les peuples de l'univers, réunie sous un même nom et dans la même souffle sinaïtique. (Trigano, 308)

Et Herzl paraît exclu de cet universalisme - peut-être à tort ? 


\section{BIBLIOGRAPHIE}

Barthes, Roland : S/Z [1970], Paris : Seuil (Points), 1976.

Barthes, Roland : «L'Ancienne Rhétorique. Aide-mémoire », ds. : Recherches rhétoriques (= Communications, 16), Paris : Seuil (points essais), 1994, 256-340.

Beckett, Samuel : Fin de partie, Paris : Minuit 1957.

Bloch, Ernst : Das Prinzip Hoffnung [1959], Frankfurt a.M. : Suhrkamp (stw) 1985 (=Werkausgabe 5)

[fr. : Le Principe Espérance II.4. Les épures d'un monde meilleur, traduit par Françoise Wuilmart, Paris : Galllimard 1982].

Blumenberg, Hans : Lebenszeit und Weltzeit, Frankfurt a.M. : Suhrkamp 1986.

Cohen, Hermann : Religion der Vernunft aus den Quellen des Judentums. Eine jüdische Religionsphilosophie, Berlin ${ }^{2} 1928$ [repr. Wiesbaden : Fourier $\left.{ }^{3} 1995\right]$.

Finkielkraut, Alain : Le Juif imaginaire, Paris : Seuil (points), 1983.

Genette, Gérard : Palimpsestes. La Littérature au second degré [1982], Paris : Seuil (points), 1992.

Giniewski : « Theodor Herzl, prophète », ds. : Herzl, Theodor : Le Pays ancien-nouveau, op. cit., 7-26.

Haddad, Gérard : Les Folies millénaristes. Les Biblioclastes, Paris : Le Livre de Poche (biblio essais), 2002.

Herzl, Theodor : Der Judenstaat : Versuch einer modernen Lösung der Judenfrage [1896] mit einem Nachwort von Henryk M. Broder, Augsburg : Ölbaum 1996 [fr. : L'État des juifs, traduit par Claude Klein, Paris : Éditions de la découverte (textes à l'appui /histoire contemporaine) 1990, première édition française : L'État juif, ds. La Nouvelle Revue internationale 1896/97]

Herzl, Theodor : Altneuland [1902], Berlin : B. Harz s.d. ; repr. http://gallica. bnf.fr [fr. : Le Pays ancien-nouveau, traduit par Paul Giniewski, Paris : Stock 1998].

Jonas, Hans : Das Prinzip Verantwortung. Versuch einer Ethik für die technologische Zivilisation [1979], Frankfurt a.M. : Suhrkamp (st) 1984.

Klein, Claude : « Essai sur le sionisme », ds. : Herzl, Thodor : L'État des juifs, op. cit., 117-186.

Klein, Claude : " Note sur les différentes éditions du Judenstaat et sur la présente traduction », ds. : Herzl, Thodor : L'État des juifs, op. cit., 5-12.

Kristeva, Julia : Séméiotikè. Recherches pour une sémanalyse [1969], Paris : Seuil (points / extraits) 1978.

Kuhnle, Till R. : « Ernst und Revolte. Ein Versuch zum existentiellen Messianismus ». In : Romanistische Zeitschrift für Literaturgeschichte / Cahiers d'Histoire des Littératures Romanes XXII.1/2, Heidelberg : Winter 1998, 107-136.

Kuhnle, Till R. : « Pas de panique ? - Apocalypse et messianisme chez Hermann Broch ». In : Germanica XXIV. Bible et littérature de langue allemande au XXe siècle, Lille : Université Charles-deGaulle (Lille III) 1999, 157-175.

Lévy, Bernard-Henri : Mémoire vive. Questions de principe VII. 1994-1995, Paris : Livre de Poche (biblio/essais), 2001. 
Löwy, Michel : Rédemption et Utopie. Le Judaïsme libertaire en Europe centrale. Une étude d'affinité élective, Paris : PUF 1988.

Mannheim, Karl : Ideologie und Utopie [1929], Frankfurt a. M. : Vittorio Klostermann ${ }^{8} 1995$.

Mercier, Louis Sébastien : L'An 2440. Rêve s'il en fut jamais [1774], Paris : La Découverte (poche), 1999.

Meschonnic, Henri : L'Utopie juive, Paris : Midrash/Desclée de Brouwer (Essais) 2001.

Sartre, Jean-Paul : Réflexions sur la question juive [1954], Paris : Gallimard (folio essais), 1993.

Triango, Shmuel : La nouvelle Question juive [1979], Paris : Gallimard (folio essais), 2002.

Verne, Jules : Vingt mille Lieues sous les mers [1869], Paris : Le Livre de Poche 1990.

Verne, Jules : L'T̂le mystérieuse [1874], Paris : Le Livre de Poche (2 Bde.) s.d.

Verne, Jules : Les 500 millions de la Bégum [1879], Paris : Le Livre de Poche, s.d.

Verne, Jules : Robur le conquérant [1886], Paris : Le Livre de Poche, s.d.

Verne, Jules : L'île à hélice [1895], Paris : U.G. E (10/18) 1978.

Verne, Jules : Paris au XX ${ }^{e}$ siècle, Paris : Hachette/Le Cherche-midi/Le Livre de Poche 1994.

\section{RÉSUMÉS}

"Si vous le voulez, ce ne sera pas un rêve " - c'est le slogan mis en exergue du roman Altneuland (fr. Terre ancienne - terre nouvelle ou Le Pays ancien-nouveau), paru en 1902, dans lequel Herzl développe le projet sioniste sous forme d'une fiction politique: cette société juive en Palestine, basée sur un système coopératif et mutualiste, qu'il a esquissée, en 1896, dans son essai Der Judenstaat. Par son réalisme technique et économique, Herzl reprend l'idéologie d'une bourgeoisie qui a abandonné l'idée de la perfectibilité du genre humain tout en gardant confiance en l'idée du progrès scientifique. Herzl pose son projet comme réalisable: «En raison du changement minime apporté au système économique dans l'État juif modèle, cette utopie n'était pas installée dans un avenir très éloigné : elle se déclare compte rendu de l'année 1920 » (E. Bloch). En effet, Herzl fait preuve d'une vision particulière de la destinée des juifs européens : l'idée de l'assimilation aux sociétés européennes qui ne signifiait qu'une pure imitation de leurs valeurs est remplacée par celle de l'émulation (dans le sens rhétorique du terme, lat. aemulatio) dans l'accomplissement de la civilisation par ces valeurs. Dans une certaine mesure, il pratique la « ré-écriture » idéologique d'une vision du monde telle qu'elle a été exprimée, entre autres, par Jules Verne. Il récuse toute philosophie de l'Histoire eschatologique, voire apocalyptique, qui - à l'instar des millénarismes chrétiens et judaïques ou de la révolution marxiste - annonce une rupture imminente : l'anéantissement du vieux monde pour faire naître sur ses décombres une ère (messianique) nouvelle. Et pourtant, le rôle attribué par Herzl au peuple juif est celui de l'avant-garde de l'humanité : «Le monde sera libre par notre liberté, enrichi de notre richesse, agrandi de notre grandeur. Et ce que nous tenterons là-bas pour notre propre prospérité aura des effets puissants et heureux pour le bien-être de l'humanité tout entière "( Der Judenstaat). Aujourd'hui, l'œuvre de Herzl paraît oubliée. Pour Shmuel Trigano, le sionisme de 1948 «a peu à voir avec le sionisme fondateur. C'est une idéologie désespérée. Elle est devenue l'idéologie de l'exil ». En fait, il ne s'agit que de la « ré-écriture » d'un blanc, car les écrits de Herzl, pourtant mineurs en tant que textes littéraires, sont toujours présents dans tout discours portant sur l'identité juive face au défi nommé Israël. 
« Wenn Ihr wollt, dann wird es kein Traum bleiben »- mit diesem Motto beginnt Theodor Herzls 1902 erschienener Roman Altneuland. Es ist die Wiederaufnahme des Der Judenstaat entworfene Programm eines jüdischen Staates in Palästina in Gestalt einer literarischen Utopie. Grundlage dieses neuen Staat soll eine nach genossenschaftlichen Prinzipien organisierte Gesellschaft sein, in der sich jeder Bürger frei entfalten könne. Der ökonomische und technische Realismus Herzls schreibt sich in die Ideologie eines Bürgertums ein, das sich von der Vorstellung eines immerfort zu Höherem strebenden Menschengeschlechts verabschiedet hat und nunmehr einzig dem wissenschaftlichen Fortschritt traut. Für Herzl ist daher die baldige Verwirklichung des Projekts Judenstaat/Altneuland durchaus denkbar: "Der geringen ökonomischen Veränderungen im jüdischen Musterstaat entsprechend, wird diese Utopie nicht weit in die Zukunft verlegt : sie gibt sich als Bericht aus dem Jahr $1920 »$ (E. Bloch). In der Tat vertritt Herzl eine besondere Vorstellung von der Bestimmung der europäischen Juden: Das Prinzip der Assimilation, der Nachahmung (imitatio) europäischer Werte, wird abgelöst von der Idee eines friedlichen Wettstreits (lat. /rhet. aemulatio) bei der Vollendung der Zivilisation durch diese Werte. In gewisser Hinsicht unternimmt hierbei Herzl eine ideologische ré-écriture (das Schreiben in Topoi, die bruchlos in einen "neuen» Text eingehen) einer Weltanschauung, wie sie etwa auch von einem Jules Verne vertreten wurde. Herzl lehnt eine eschatologische oder apokalyptische Geschichtsphilosophie ab, die - in der Tradition eines jüdisch-christlichen oder säkularen Millenarimus - eine bevorstehende Zäsur verkündet : die Vernichtung der alten Welt, damit auf diese ein neues (messianisches) Zeitalter folge. Dennoch sieht Herzl im jüdischen Volk die Avantgarde der Menschheit: "Die Welt wird durch unsere Freiheit befreit, durch unseren Reichtum bereichert, und vergrößert durch unserer Größe. Und was wir dort [in Palästina] nur für unser eigenes Gedeihen versuchen, wirkt machtvoll und beglückend hinaus zum Wohle aller Menschen » (Der Judenstaat). Heute scheint das Werk Herzls vergessen. Für Shmuel Trigano etwa hat der Zionismus von 1948 nur noch wenig gemein mit dem Zionismus seiner Gründerväter. Dieser sei eine Ideologie der Verzweiflung, eine Ideologie des Exils. Dennoch sind die literarisch wenig anspruchsvollen Schriften Herzls stets gegenwärtig. Angesichts der Herausforderung durch die Existenz des Staates Israel, werden sie - quasi als ré-écriture einer Leerstelle (blanc) fortwährend um- und weitergeschrieben.

\section{AUTEUR}

\section{TILL R. KUHNLE}

Universität Augsburg 